\title{
On Combinatorics of Quiver Component Formulas
}

\author{
ALEXANDER YONG \\ ayong@math.berkeley.edu \\ Department of Mathematics, University of California, Berkeley, 970 Evans Hall, Berkeley, CA 94702-3840, USA
}

Received July 1, 2003; Revised August 12, 2004; Accepted September 8, 2004

\begin{abstract}
Buch and Fulton [9] conjectured the nonnegativity of the quiver coefficients appearing in their formula for a quiver cycle. Knutson, Miller and Shimozono [24] proved this conjecture as an immediate consequence of their "component formula". We present an alternative proof of the component formula by substituting combinatorics for Gröbner degeneration [23, 24]. We relate the component formula to the work of Buch, Kresch, Tamvakis and the author [10] where a "splitting" formula for Schubert polynomials in terms of quiver coefficients was obtained. We prove analogues of this latter result for the type $B C D$-Schubert polynomials of Billey and Haiman [4]. The form of these analogues indicate that it should be interesting to pursue a geometric context that explains
\end{abstract} them.

Keywords: degeneracy loci, quiver polynomials, component formula, generalized Littlewood-Richardson coefficients

\section{Introduction}

Buch and Fulton [9] established a formula for a general kind of degeneracy locus associated to an oriented quiver of type $A$. This formula is in terms of Schur polynomials and certain integers, the quiver coefficients, which generalize the classical Littlewood-Richardson coefficients. Buch and Fulton further conjectured the nonnegativity of these quiver coefficients, and this conjecture was recently proved by Knutson, Miller and Shimozono [24]. In fact, they obtained a stronger result, the "component formula", whose proof was based on combinatorics, a "ratio formula" derived from a geometric construction due to Zelevinsky [32] and the method of Gröbner degeneration, applying multidegree formulae for matrix Schubert varieties from [23].

In this paper, we report on some combinatorial aspects of this story. In the first "half", we prove a combinatorial result (Theorem 1) that replaces the Gröbner degeneration part of their argument. This allows for an entirely combinatorial proof of the component formula from the ratio formula. The utility of this approach goes beyond merely satisfying the desire to have a combinatorial solution to a combinatorial problem. It appears difficult to extend the Gröbner degeneration approach to give a $K$-theoretic generalization of the component formula necessary to prove the main "alternating signs" conjecture of [7]. Therefore, alternatives to the Gröbner degeneration approach became necessary. This part of our paper was written in response to this need. In fact, after this paper was submitted, the combinatorial approach given by Theorem 1 was naturally generalized for this purpose, see [8, p. 11] and $[31$, p. 2]. 
While the first half concerns applying combinatorics to further understand geometry, our second half builds towards a geometric project suggested by combinatorics. The component formula is connected to the work of Buch, Kresch, Tamvakis and the author [10], where a formula was obtained for Fulton's universal Schubert polynomials [19] (see the Appendix where this connection is made precise via a bijection). In [10], this formula was used to obtain a "splitting" formula for the ordinary Schubert polynomials of Lascoux and Schützenberger [27] in terms of quiver coefficients. We provide analogues of this splitting formula for the type $B C D$-Schubert polynomials of Billey and Haiman [4], in terms of a new collection of positive combinatorial coefficients that appear combinatorially analogous to the quiver coefficients. The geometry that underlies these formulas remains unclear. However, their shape is very suggestive. It should be interesting to pursue a geometric (degeneracy locus/Schubert calculus) setting that explains these formulas and the positivity.

Let $\mathfrak{X}$ be a nonsingular complex variety and $E_{0} \rightarrow E_{1} \rightarrow \cdots \rightarrow E_{n}$ a sequence of vector bundles and bundle maps over $\mathfrak{X}$. A set of rank conditions for this sequence is a collection of nonnegative integers $\mathbf{r}=\left\{r_{i j}\right\}$ for $0 \leq i \leq j \leq n$. This data defines a degeneracy locus in $\mathfrak{X}$,

$$
\Omega_{\mathbf{r}}\left(E_{\bullet}\right)=\left\{x \in \mathfrak{X} \mid \operatorname{rank}\left(E_{i}(x) \rightarrow E_{j}(x)\right) \leq r_{i j}, \forall i<j\right\},
$$

where $r_{i i}$ is by convention the rank of the bundle $E_{i}$. We require that the rank conditions $\mathbf{r}$ occur, i.e., there exists a sequence of vector spaces and linear maps $V_{0} \rightarrow V_{1} \rightarrow \cdots \rightarrow V_{n}$ such that $\operatorname{dim}\left(V_{i}\right)=r_{i i}$ and $\operatorname{rank}\left(V_{i} \rightarrow V_{j}\right)=r_{i j}$. This is known to be equivalent to $r_{i j} \leq \min \left(r_{i, j-1}, r_{i+1, j}\right)$ for $i<j$ and $r_{i j}-r_{i-1, j}-r_{i, j+1}+r_{i-1, j+1} \geq 0$ for $0 \leq i \leq j \leq n$ where $r_{i j}=0$ if $i$ or $j$ are not between 0 and $n$.

The expected (and maximal) codimension of the locus $\Omega_{\mathbf{r}}\left(E_{\bullet}\right)$ in $\mathfrak{X}$ is

$$
d(\mathbf{r})=\sum_{i<j}\left(r_{i, j-1}-r_{i j}\right) \cdot\left(r_{i+1, j}-r_{i j}\right)
$$

Buch and Fulton [9] gave a formula for the cohomology class of the quiver cycle $\left[\Omega_{\mathbf{r}}\left(E_{\bullet}\right)\right]$ in $\mathrm{H}^{*}(\mathfrak{X})$, assuming it has this codimension:

$$
\left[\Omega_{\mathbf{r}}\left(E_{\bullet}\right)\right]=\sum_{\underline{\mu}} c_{\underline{\mu}}(\mathbf{r}) s_{\mu_{1}}\left(E_{0}-E_{1}\right) \cdots s_{\mu_{n}}\left(E_{n-1}-E_{n}\right) .
$$

Here the sum is over sequences of partitions $\mu=\left(\mu_{1}, \ldots, \mu_{n}\right)$, each $s_{\mu_{i}}$ is a super-symmetric Schur function in the Chern roots of the bundles in its argument, and the quiver coefficients $c_{\mu}(\mathbf{r})$ are integers, conjectured to be nonnegative by Buch and Fulton. These coefficients are among the most interesting Schubert calculus numbers that we presently know formulas for, see, e.g., $[6,9,10,12]$ and the references therein. 
This Buch-Fulton conjecture was recently proved by Knutson, Miller and Shimozono [24]. In fact, they prove the following "component formula":

$$
\left[\Omega_{\mathbf{r}}\left(E_{\bullet}\right)\right]=\sum_{W \in W_{\min }(\mathbf{r})} F_{w_{1}}\left(E_{0}-E_{1}\right) \cdots F_{w_{n}}\left(E_{n-1}-E_{n}\right)
$$

where $W_{\min }(\mathbf{r})$ is the set of minimal length "lacing diagrams" for $\mathbf{r}$, and each $F_{w_{i}}$ is a double Stanley symmetric function. The nonnegativity of the quiver coefficients (and a positive combinatorial interpretation for what they count) follows immediately from (3) by using a formula for the expansion of a Stanley symmetric function into a positive sum of Schur functions [14, 28].

The proof of (3) in [24] uses the new "ratio formula" for $\left[\Omega_{\mathbf{r}}\left(E_{\bullet}\right)\right]$, which is derived from an alternate form of a geometric construction originally due to Zelevinsky [32] and developed scheme-theoretically by Lakshmibai and Magyar [25], for details, see Section 3.3. The proof proceeds by utilizing combinatorics to derive an intermediate formula for $\left[\Omega_{\mathbf{r}}\left(E_{\bullet}\right)\right]$ as a multiplicity-free sum of products of Stanley functions over some minimal length lacing diagrams for $\mathbf{r}$. Then Gröbner degeneration [23, 24] is used to prove that all minimal length lacing diagrams for $\mathbf{r}$ actually appear.

Our aforementioned Theorem 1 (Section 2) is an explicit injection of $W_{\min }(\mathbf{r})$ into $\mathcal{R} C(v(\mathbf{r}))$, the set of RC-graphs for the "Zelevinsky permutation" of $\mathbf{r}$. When substituted for the Gröbner degeneration part of this proof of (3) and combined with the rest of [24], this provides a purely combinatorial derivation of the component formula (3) from the ratio formula, this is explained in Section 3.

The formula for the universal Schubert polynomials obtained in [10] was applied there to prove a "splitting" formula for the ordinary Schubert polynomials [27] in terms of quiver coefficients. In Section 4, we obtain the analogues of this splitting formula for the type $B C D$-Schubert polynomials of Billey and Haiman [4] and introduce a collection of positive combinatorial coefficients that appear combinatorially analogous to the quiver coefficients. We also remark on some of the expected geometric features of these coefficients.

In the Appendix, we provide a bijection between the labeling set in the righthand side of (3) when the rank conditions are determined by a permutation, and its counterpart in the formula for Fulton's universal Schubert polynomials obtained by Buch, Kresch, Tamvakis and the author [10]. This explains how the component formula generalizes the aforementioned formula of [10].

We thank Sergey Fomin and Ezra Miller for their questions that initiated this work. We are extremely grateful to Ezra Miller for introducing us to the results in [24] and for his many helpful comments, including suggesting a simplification in the proof of Theorem 1 and providing macros for drawing RC-graphs and pipe dreams. We would also like to thank Anders Buch, Harm Derksen, Bill Fulton, Andrew Kresch, John Stembridge, Harry Tamvakis and the referees for helpful comments. 


\section{Embedding lacing diagrams into RC-graphs}

\subsection{Ranks and laces}

Let $\mathbf{r}=\left\{r_{i j}\right\}$ for $0 \leq i \leq j \leq n$ be a set of rank conditions. It is convenient to arrange them in a rank diagram [9]:

$$
\begin{array}{ccccccccc}
E_{0} & \rightarrow & E_{1} & \rightarrow & E_{2} & \rightarrow & \cdots & \rightarrow & E_{n} \\
r_{00} & & r_{11} & & r_{22} & & \ldots & & r_{n n} \\
& r_{01} & & r_{12} & & \cdots & & r_{n-1, n} & \\
& & r_{02} & & \ldots & & r_{n-2, n} & & \\
& & & \ddots & & & & & \\
& & & & & & & & \\
& & & & & & & & \\
& & & & & & & &
\end{array}
$$

We will need some notation and terminology introduced in [24]. The lace array $s(\mathbf{r})$ is defined by

$$
s_{i j}(\mathbf{r})=r_{i j}-r_{i-1, j}-r_{i, j+1}+r_{i-1, j+1},
$$

for $0 \leq i \leq j \leq n$, where as before, $r_{i j}=0$ if $i$ or $j$ are not between 0 and $n$. Note that each entry of $s_{i j}(\mathbf{r})$ is nonnegative, by our assumptions on $\mathbf{r}$. A lacing diagram $W$ is a graph on $r_{00}+\cdots+r_{n n}$ vertices arranged in $n$ bottom-justified columns labeled from 0 to $n$. The $i$ th column consists of $r_{i i}$ vertices. The edges of $W$ connect consecutive columns in such a way that no two edges connecting two given columns share a vertex. A lace is a connected component of such a graph and an $(i, j)$-lace starts in column $i$ and ends in column $j$. Also, $W$ is a lacing diagram for $\mathbf{r}$ if the number of $(i, j)$-laces equals $s_{i j}(\mathbf{r})$.

Example 1 For $n=3$, the rank conditions

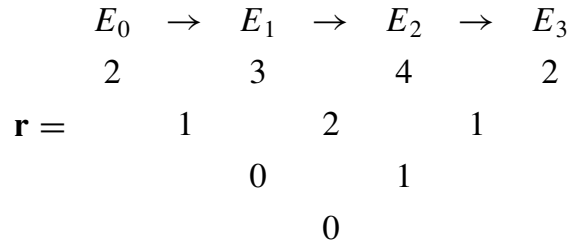


give

$s(\mathbf{r})=$\begin{tabular}{llll|l}
3 & 2 & 1 & 0 & $i / j$ \\
\hline & & & 1 & 0 \\
& & 0 & 1 & 1 \\
& 2 & 1 & 0 & 2 \\
& 0 & 1 & 0 & 3
\end{tabular}

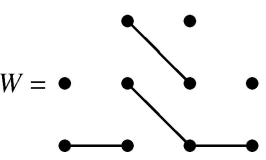

Each lacing diagram $W$ corresponds to an ordered $n$-tuple $\left(w_{1}, w_{2}, \ldots, w_{n}\right)$ of partial permutations, where $w_{i}$ is represented by the $r_{i-1} \times r_{i}(0,1)$-matrix with an entry 1 in position $(\alpha, \beta)$ if and only if an edge connects the $\alpha$ th vertex in column $i-1$ (counting from the bottom) to the $\beta$ th vertex in column $i$. For example, the lacing diagram $W$ from Example 1 corresponds to:

$$
\left(\left(\begin{array}{lll}
1 & 0 & 0 \\
0 & 0 & 0
\end{array}\right),\left(\begin{array}{llll}
0 & 0 & 0 & 0 \\
1 & 0 & 0 & 0 \\
0 & 1 & 0 & 0
\end{array}\right),\left(\begin{array}{ll}
1 & 0 \\
0 & 0 \\
0 & 0 \\
0 & 0
\end{array}\right)\right)
$$

Any $a \times b$ partial permutation $\rho$ has a minimal length embedding $\tilde{\rho}$ in the symmetric group $S_{a+b}$. The permutation matrix for $\tilde{\rho}$ is constructed to have $\rho$ as a northwest submatrix. In the columns of $\tilde{\rho}$ to the right of $\rho$, place a 1 in each of the top $a$ rows for which $\rho$ does not already have one, making sure that the new 1's progress from northwest to southeast. Similarly, in every row of $\tilde{\rho}$ below $\rho$, place 1 's going northwest to southeast, in those columns which do not have one yet. For example, the following are the minimal length embeddings of the above partial permutations:

$$
\left(\left(\begin{array}{lllll}
1 & 0 & 0 & 0 & 0 \\
0 & 0 & 0 & 1 & 0 \\
0 & 1 & 0 & 0 & 0 \\
0 & 0 & 1 & 0 & 0 \\
0 & 0 & 0 & 0 & 1
\end{array}\right),\left(\begin{array}{lllllll}
0 & 0 & 0 & 0 & 1 & 0 & 0 \\
1 & 0 & 0 & 0 & 0 & 0 & 0 \\
0 & 1 & 0 & 0 & 0 & 0 & 0 \\
0 & 0 & 1 & 0 & 0 & 0 & 0 \\
0 & 0 & 0 & 1 & 0 & 0 & 0 \\
0 & 0 & 0 & 0 & 0 & 1 & 0 \\
0 & 0 & 0 & 0 & 0 & 0 & 1
\end{array}\right),\left(\begin{array}{llllll}
1 & 0 & 0 & 0 & 0 & 0 \\
0 & 0 & 1 & 0 & 0 & 0 \\
0 & 0 & 0 & 1 & 0 & 0 \\
0 & 0 & 0 & 0 & 1 & 0 \\
0 & 1 & 0 & 0 & 0 & 0 \\
0 & 0 & 0 & 0 & 0 & 1
\end{array}\right)\right) .
$$

We define the length of a partial permutation matrix $\rho$ to be equal to $\ell(\tilde{\rho})$. Here $\ell(\tilde{\rho})$ is the length of $\tilde{\rho}$, the smallest number $\ell$ for which $\tilde{\rho}$ can be written as a product of $\ell$ simple transpositions. The length of a lacing diagram $W=\left(w_{1}, w_{2}, \ldots, w_{n}\right)$ is denoted $\ell(W)$, where $\ell(W)=\ell\left(w_{1}\right)+\ell\left(w_{2}\right)+\cdots+\ell\left(w_{n}\right)$. A lacing diagram $W$ for $\mathbf{r}$ is a minimal length lacing diagram if $\ell(W)=d(\mathbf{r})$. For instance, the lacing diagram $W$ in 
Example 1 is of minimal length. We denote the set of minimal length lacing diagrams for $\mathbf{r}$ by $W_{\min }(\mathbf{r})$.

\subsection{The Zelevinsky permutation}

Also associated to $\mathbf{r}$ is the Zelevinsky permutation $v(\mathbf{r}) \in S_{d}$, where $d=r_{00}+r_{11}+\cdots+r_{n n}$ [24]. This is defined via its graph $G(v(\mathbf{r}))$, the collection of the $d^{2}$ points $\{(i, w(i))\}_{1 \leq i \leq d}$ in $d \times d=[1, d] \times[1, d]$.

Partition the $d \times d$ box into $(n+1)^{2}$ blocks $\left\{M_{i j}\right\}$ for $0 \leq i, j \leq n$, read as in block matrix form; so $M_{i j}$ has dimension $r_{i i} \times r_{n-j, n-j}$ (later, we will also need the sets $H_{j}=\bigcup_{i=0}^{n} M_{i j}$ and $V_{i}=\bigcup_{j=0}^{n} M_{i j}$ of horizontal and vertical strips respectively). Beginning with $M_{n n}$ and continuing right to left and bottom up, place $s_{n-j, i}(\mathbf{r})$ points into the block $M_{i j}$, as southeast as possible such that no two points lie in the same row or column (in particular, points go northwest-southeast in each block). Complete the empty rows and columns by placing points in the super-antidiagonal blocks $M_{i, n-i-1}, i=0, \ldots, n-1$. In general, this concluding step is achieved by placing points contiguously on the main diagonal of each of the super-antidiagonal blocks. That this procedure produces a permutation matrix is proved in [24].

Later we will need the fact that

$$
\ell(v(r))=\left|\bigcup_{i+j \leq n-2} M_{i j}\right|+d(\mathbf{r}) .
$$

This follows from [24, Section 1.2] but can also be directly verified from (1) and (4).

Example 2 For the rank conditions $\mathbf{r}$ from Example 1, we obtain

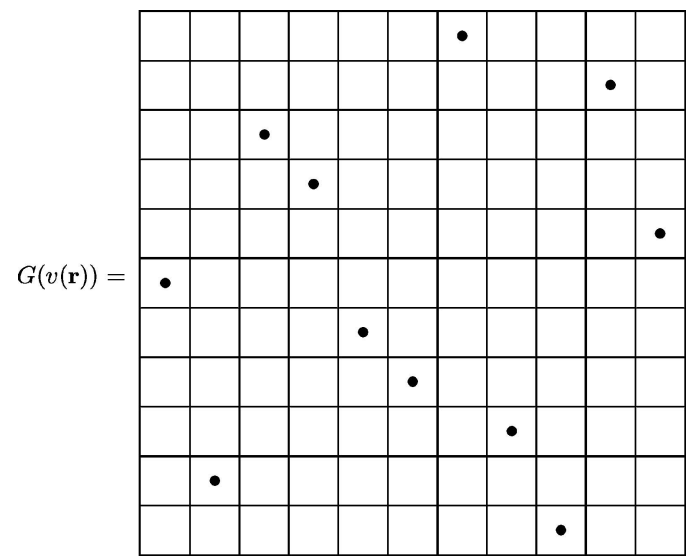


Thus,

$$
v(\mathbf{r})=\left(\begin{array}{ccccccccccc}
1 & 2 & 3 & 4 & 5 & 6 & 7 & 8 & 9 & 10 & 11 \\
7 & 10 & 3 & 4 & 11 & 1 & 5 & 6 & 8 & 2 & 9
\end{array}\right)
$$

\subsection{RC-graphs}

We continue by recalling the definition of the set $\mathcal{R} C(w)$ of $\mathrm{RC}$-graphs for a permutation $w \in S_{d}[2,18]$. For positive integers $a$ and $b$, consider the $a \times b$ square grid with the box in row $i$ and column $j$ labeled $(i, j)$ as in an $a \times b$ matrix. Tile the grid so that each box either contains a cross - or an elbow joint $r$. Thus the tiling appears as a "network of pipes". Such a tiled grid is a pipe dream [23].

A pipe network for $w$ is a pipe dream where $a=b=d$, no crosses appear in the lower triangular part of the grid and the pipe entering at row $i$ exits at column $w(i){ }^{1}$ Finally, the set $\mathcal{R} C(w)$ of RC-graphs for a permutation $w \in S_{d}$ is the set of pipe networks for $w$ such that any two pipes cross at most once. We omit drawing the "sea of waves" that appear in the southeast triangle of an RC-graph.

Each RC-graph is known to encode a reduced word for $w$. Let $u_{1} u_{2} \cdots u_{\ell(w)}$ be a reduced word for $w$. Then a sequence $\left(\mu_{1}, \mu_{2}, \ldots, \mu_{\ell(w)}\right)$ is a reduced compatible sequence for $w$ if it satisfies

- $\mu_{1} \leq \mu_{2} \leq \cdots \leq \mu_{\ell(w)}$

- $\mu_{j} \leq u_{j}$ for $1 \leq j \leq \ell(w)$

- $\mu_{j}<\mu_{j+1}$ if $u_{j}<u_{j+1}$

The following fact follows from the definition of an RC-graph:

Proposition 1 ([2]) If $\left(\mu_{1}, \ldots, \mu_{\ell(w)}\right)$ is a reduced compatible sequence for $w$, then the pipe dream with crosses at $\left(\mu_{k}, u_{k}-\mu_{k}+1\right)$ for $1 \leq k \leq \ell(w)$ and elbow joints elsewhere, is an $R C$-graph for $w$.

\subsection{Main result}

Let $W=\left(w_{1}, w_{2}, \ldots, w_{n}\right)$ be a lacing diagram and fix $\mathbf{r}=\left\{r_{i j}\right\}, 0 \leq i \leq j \leq n$. A pipe network $R$ for $w$ maps to $W$ (denoted $\Phi(R)=W$ ) if for all $1 \leq k \leq n, 1 \leq s \leq r_{k-1, k-1}$ and $1 \leq t \leq r_{k k}$, a pipe enters at the top of the box

$$
\left(r_{00}+r_{11}+\cdots+r_{k-2, k-2}+1, r_{n n}+r_{n-1, n-1}+\cdots+r_{k-1, k-1}-s+1\right)
$$

and exits at the bottom of the box

$$
\left(r_{00}+r_{11}+\cdots+r_{k-1, k-1}, r_{n n}+r_{n-1, n-1}+\cdots+r_{k k}-t+1\right)
$$




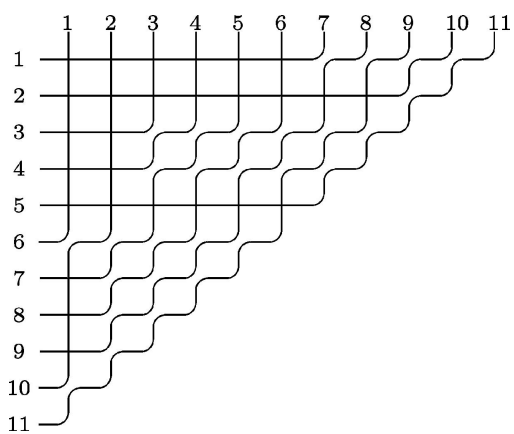

Figure 1. An RC-graph for $v(\mathbf{r})$ from Example 2.

if and only if the $(s, t)$ entry of the partial permutation matrix $w_{k}$ equals 1 . Here, we set $r_{k k}=0$ if $k<0$. In other words, $\Phi(R)=W$ if the above pipes correspond to the laces of $W$. For example, the RC-graph for $v(\mathbf{r})$ in figure 1 maps to the lacing diagram $W$ from Example 1. This can be seen in the picture below: straightening the (partial) pipes of $W$ and right-justifying the result gives $W$, after reflecting across a northwest-southeast diagonal.

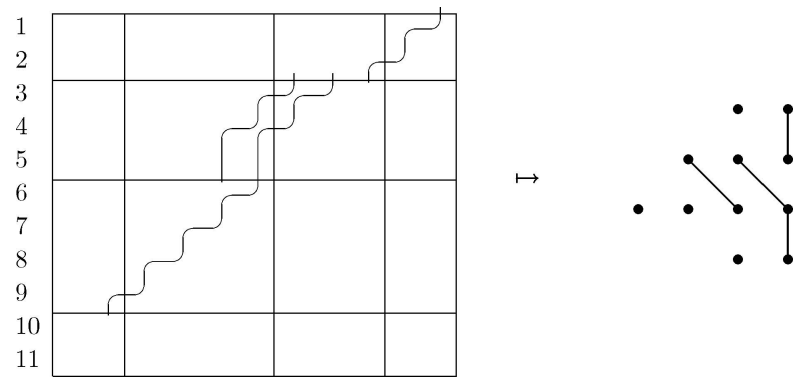

The following is our main result:

Theorem 1 Let $\mathbf{r}=\left\{r_{i j}\right\}$ for $0 \leq i \leq j \leq n$ be a set of rank conditions. There is an explicit injection from $W_{\min }(\mathbf{r}) \hookrightarrow \mathcal{R} C(v(\mathbf{r}))$ sending $W \mapsto D$ such that $\Phi(D)=W$.

As explained in Section 3, combinatorics combined with the ratio formula gives the following variation of (3):

$$
\left[\Omega_{\mathbf{r}}\left(E_{\bullet}\right)\right]=\sum_{W \in W_{R P}(\mathbf{r})} F_{w_{1}}\left(E_{0}-E_{1}\right) \cdots F_{w_{n}}\left(E_{n-1}-E_{n}\right),
$$

where $W_{R P}(\mathbf{r})$ are those $W \in W_{\min }(\mathbf{r})$ for which there is a $D \in \mathcal{R} C(v(\mathbf{r}))$ such that $\Phi(D)=W$. Thus, Theorem 1 supplies the missing ingredient for a combinatorial derivation of (3) from the ratio formula. 


\subsection{Proof of Theorem 1}

Let $S_{d}(\mathbf{r})$ denote the set of permutations $w$ in $S_{d}$ such that $G(w)$ contains the same number of points in $M_{i j}$ as $G(v(\mathbf{r}))$ does, for all $0 \leq i, j \leq n$. Our proof of Theorem 1 uses the following:

Proposition 2 Let $\mathbf{r}=\left\{r_{i j}\right\}$ for $0 \leq i \leq j \leq n$ be a set of rank conditions and let $w \in S_{d}$, $d=r_{00}+r_{11}+\cdots+r_{n n}$. The following are equivalent:

(I) $w=v(\mathbf{r})$;

(II) $w$ is the minimal length element of $S_{d}(\mathbf{r})$;

(III) there exists a pipe network $D$ for $w$ and there exists a lacing diagram $W$ for $\mathbf{r}$ such that $D$ has every box in $\bigcup_{i+j \leq n-2} M_{i j}$ tiled by crosses, $\Phi(D)=W$, and $\ell(w) \leq \ell(v(\mathbf{r}))$.

Proof: The length of $w \in S_{d}(\mathbf{r})$ is computed from $G(w)$ by counting those pairs of dots where one is situated to the northeast of the other. Call such a pair unavoidable if the dots actually appear in blocks where one is situated (strictly) northeast of the other. The number of unavoidable pairs is constant on $S_{d}(\mathbf{r})$. Moreover, observe that all of the pairs contributing to the length of $v(\mathbf{r})$ are unavoidable. On the other hand, if $w \neq v(\mathbf{r})$, then at least one pair contributing to $\ell(w)$ is not unavoidable. Thus (I) is equivalent to (II).

That (I) implies (III) is immediate from [24, Theorem 5.10], but we include a proof for completeness. Take any $D \in \mathcal{R} C(v(\mathbf{r}))$. The definition of $v(\mathbf{r})$ implies that $D$ has every box in $\bigcup_{i+j \leq n-2} M_{i j}$ tiled by crosses. Moreover, $D$ gives a lacing diagram $W$ such that $\Phi(D)=W$. Observe that the number of pipes of $D$ that enter in the $i$ th horizontal strip and exit in the $j$ th vertical strip is equal to the number of points of $G(v(\mathbf{r}))$ in $M_{i j}$ for $0 \leq i, j \leq n$. From this and the definition of $v(\mathbf{r})$ it follows that $W$ is in fact a lacing diagram for $\mathbf{r}$.

Finally, suppose (III) holds. By considering where the pipes of $D$ go in relation to $W$, one finds that $G(w)$ and $G(v(\mathbf{r}))$ have the same number of points in any block on the main anti-diagonal and below, i.e., blocks $M_{i j}$ where $i+j \geq n$. The condition on the boxes of $\bigcup_{i+j \leq n-2} M_{i j}$ implies that the only other points of $G(w)$ appear in the blocks $M_{i, n-i-1}$, $0 \leq i \leq n-1$ on the super-antidiagonal. Since $w$ is a permutation, each of these blocks must have the same number of points as its counterpart in $G(v(\mathbf{r}))$, i.e. $w \in S_{d}(\mathbf{r})$. Since we already know $v(\mathbf{r})$ is the unique minimal length element of $S_{d}(\mathbf{r})$, the assumption that $\ell(w) \leq \ell(v(\mathbf{r}))$ implies (II).

Let $\rho$ be a partial permutation represented by an $a \times b$ matrix. Consider the diagram $D(\tilde{\rho})$ of $\tilde{\rho}$, which consists of the boxes $(i, j)$ in $(a+b) \times(a+b)$ such that $\tilde{\rho}(i)>j$ and $\tilde{\rho}^{-1}(j)>i$. Associated to $\tilde{\rho}$ is its canonical reduced word. This is obtained by numbering the boxes of $D(\tilde{\rho})$ consecutively in each row, from right to left, starting with the number of the row. Then the rows are read left to right, from top to bottom (see, e.g. [30, Section 2.1]).

Lemma 1 Let $u_{1} u_{2} \cdots u_{\ell(\tilde{\rho})}$ be the canonical reduced word for $\tilde{\rho}$. Then the set $\left\{k_{1}<\right.$ $\left.k_{2}<\cdots<k_{p}\right\}$ of indices $k$ where $u_{k}<u_{k+1}$ has size at most a. Moreover, $j \leq u_{k}$ for all $k \in\left[k_{j-1}+1, k_{j}\right]$, where $k_{0}=0$. 
Proof: By construction, $D(\tilde{\rho})$ sits inside the northwest $a \times b$ rectangle of the $(a+b) \times(a+b)$ box. Since the labels of the boxes in the construction of the canonical reduced word decrease from left to right along each row, there can be at most $a$ indices $k$ where $u_{k}<u_{k+1}$. The fact that each entry of the $t$ th row of the filling of $D(\tilde{\rho})$ is at least $t$ implies the remainder of the claim.

Example 3 Let $\rho$ be the partial permutation represented by the matrix:

$$
\left(\begin{array}{llll}
0 & 0 & 0 & 0 \\
1 & 0 & 0 & 0 \\
0 & 0 & 0 & 1
\end{array}\right) .
$$

The canonical reduced word for $\tilde{\rho}$ is obtained below (see figure 2).

The following fact is immediate from the main theorem of [2]. We include a proof for completeness:

Lemma 2 There exists an RC-graph for $\tilde{\rho}$ such that all crosses occur in its northwest $a \times b$ sub-rectangle.

Proof: Let $u_{1} u_{2} \cdots u_{\ell(\tilde{\rho})}$ be the canonical reduced word for $\tilde{\rho}$. By Lemma 1,

$$
(\underbrace{1,1, \ldots, 1}_{k_{1}}, \underbrace{2,2, \ldots, 2}_{k_{2}}, \ldots, \underbrace{p, p, \ldots, p}_{k_{p}})
$$

is a reduced compatible sequence for $\tilde{\rho}$, and the conclusion follows from Proposition 1.

Example 4 Continuing the previous example, the reduced compatible sequence (6) corresponding to the canonical reduced word for $\tilde{\rho}$ is

$(1,1,1,1,2,2)$.

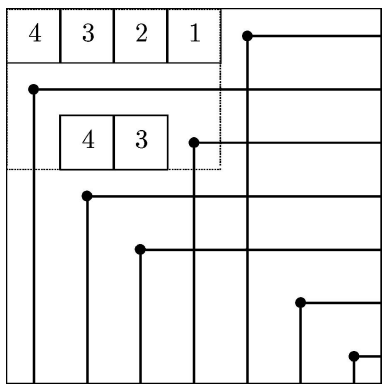

Figure 2. The canonical reduced word $4321 \cdot 43$ for $\tilde{\rho}$. 
By Proposition 1, there is an RC-graph for $\tilde{\rho}$ with crosses from

$\{(1,4),(1,3),(1,2),(1,1),(2,3),(2,2)\}$.

That RC-graph is

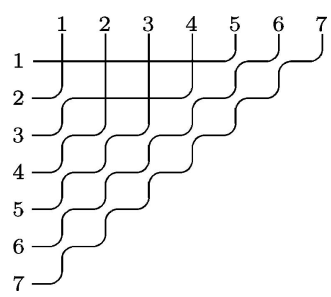

Conclusion of the Proof of Theorem 1. Construct a pipe dream $D$ starting with a $d \times d$ box as follows (see figure 3). For $k=1,2, \ldots, n$ let $D_{k}$ be the RC-graph obtained by applying Lemma 2 to the partial permutation $w_{k}$. Then let $\bar{D}_{k}$ denote the northwest $r_{k-1, k-1} \times r_{k k}$ sub-pipe dream, rotated 180 degrees. Overlay $\bar{D}_{k}$ into $M_{k-1, n-k}$. For the remaining boxes, place crosses in the top $r_{00}+r_{11}+\cdots+r_{n-2, n-2}$ rows of the $d \times d$ box and elbow joints elsewhere. This defines a pipe network $D$ for some permutation $w \in S_{d}$. By construction, $\Phi(D)=W$ and moreover, the number of crosses in $D$ is

$$
\left|\bigcup_{i+j \leq n-2} M_{i j}\right|+\ell(W)
$$

Since $W$ is minimal length, $\ell(W)=d(\mathbf{r})$ and so by (5), $l(w) \leq l(v(\mathbf{r}))$. Then by Proposition 2, $w=v(\mathbf{r})$ and thus $D \in \mathcal{R} C(v(\mathbf{r}))$. This construction describes the desired injection.

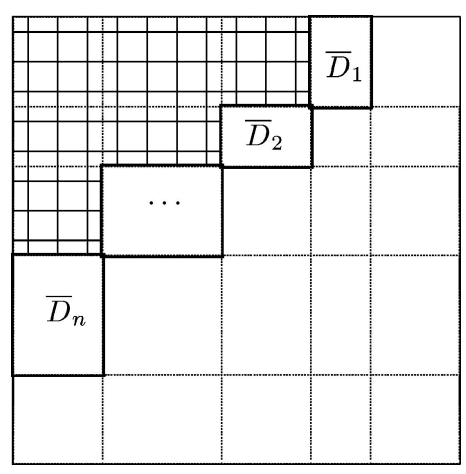

Figure 3. Construction of $D$. 
For example, the RC-graph given in figure 1 is the image of $W$ from Example 1 under the embedding map of Theorem 1 .

\section{The component formula}

\subsection{Schubert polynomials}

We begin by recalling the definition of the double Schubert polynomials of Lascoux and Schützenberger $[26,27]$. Let $X=\left(x_{1}, x_{2}, \ldots\right)$ and $Y=\left(y_{1}, y_{2}, \ldots\right)$ be two sequences of commuting independent variables. Given a permutation $w \in S_{d}$, the double Schubert polynomial $\mathfrak{S}_{w}(X ; Y)$ is defined as follows. If $w=w_{0}$ is the longest permutation in $S_{d}$ then we set

$$
\mathfrak{S}_{w_{0}}(X ; Y)=\prod_{i+j \leq d}\left(x_{i}-y_{j}\right)
$$

Otherwise there is a simple transposition $s_{i}=(i, i+1) \in S_{d}$ such that $\ell\left(w s_{i}\right)=\ell(w)+1$. We then define

$$
\mathfrak{S}_{w}(X ; Y)=\partial_{i}\left(\mathfrak{S}_{w s_{i}}(X ; Y)\right)
$$

where $\partial_{i}$ is the divided difference operator given by

$$
\partial_{i}(f)=\frac{f\left(x_{1}, \ldots, x_{i}, x_{i+1}, \ldots, x_{d}\right)-f\left(x_{1}, \ldots, x_{i+1}, x_{i}, \ldots, x_{d}\right)}{x_{i}-x_{i+1}}
$$

The (single) Schubert polynomial is defined by $\mathfrak{S}_{w}(X)=\mathfrak{S}_{w}(X ; 0)$. By convention, if $w$ is a partial permutation, we define $\mathfrak{S}_{w}=\mathfrak{S}_{\tilde{w}}$ where $\tilde{w}$ is its minimal length embedding as a permutation.

\subsection{Symmetric functions}

Let $\mathbf{x}_{\mathbf{r}}^{i}=\left(x_{1}^{i}, x_{2}^{i}, \ldots, x_{r_{i j}}^{i}\right)$ be the Chern roots of the bundle $E_{i}$ for $0 \leq i \leq n$. Then for any partition $\lambda=\left(\lambda_{1} \geq \lambda_{2} \geq \cdots \geq 0\right)$ define

$$
s_{\lambda}\left(E_{i}-E_{i+1}\right)=s_{\lambda}\left(\mathbf{x}_{\mathbf{r}}^{i}-\mathbf{x}_{\mathbf{r}}^{i+1}\right)
$$

to be a super-symmetric Schur function in these roots. We will make use of the notation $\mathbf{x}_{\mathbf{r}}=$ $\left(\mathbf{x}_{\mathbf{r}}^{0}, \ldots, \mathbf{x}_{\mathbf{r}}^{n}\right)$ and $\check{\mathbf{x}}_{\mathbf{r}}=\left(\mathbf{x}_{\mathbf{r}}^{n}, \ldots, \mathbf{x}_{\mathbf{r}}^{0}\right)$. Similarly, $\mathbf{y}_{\mathbf{r}}=\left(\mathbf{y}_{\mathbf{r}}^{n}, \ldots, \mathbf{y}_{\mathbf{r}}^{0}\right)$, where $\mathbf{y}_{\mathbf{r}}^{\mathbf{i}}=\left(y_{1}^{i}, \ldots, y_{r_{i j}}^{i}\right)$ for $0 \leq i \leq n$. We will also need collections of infinite alphabets $\mathbf{x}, \check{\mathbf{x}}$ and $\mathbf{y}$, where we set $r_{i i}=\infty$ for each $i$ in the definitions above.

For each permutation $w \in S_{d}$ there is a stable Schubert polynomial or Stanley symmetric function $F_{w}$ in $X$ which is uniquely determined by the property that

$$
F_{w}\left(x_{1}, \ldots, x_{k}, 0,0, \ldots\right)=\mathfrak{S}_{1^{m} \times w}\left(x_{1}, \ldots, x_{k}, 0,0, \ldots\right)
$$


for all $m \geq k$. Here $1^{m} \times w \in S_{d+m}$ is the permutation which is the identity on $\{1, \ldots, m\}$ and which maps $j$ to $w(j-m)+m$ for $j>m$ (see [29, (7.18)]). When $F_{w}$ is written in the basis of Schur functions, one has

$$
F_{w}=\sum_{\alpha:|\alpha|=\ell(w)} d_{w \alpha} s_{\alpha}
$$

for some nonnegative integers $d_{w \alpha}[14,28]$. This also defines the double Stanley symmetric function $F_{w}(X-Y)$.

\subsection{Combinatorics and the proof of (3)}

Let us now explain how our work from Section 3 leads to a combinatorial proof of (3). First, we summarize the development in [24]:

The double quiver polynomial is defined using the following ratio formula:

$$
Q_{\mathbf{r}}\left(\mathbf{x}_{\mathbf{r}} ; \mathbf{y}_{\mathbf{r}}\right)=\frac{\mathfrak{S}_{v(\mathbf{r})}\left(\mathbf{x}_{\mathbf{r}} ; \mathbf{y}_{\mathbf{r}}\right)}{\mathfrak{S}_{v(H o m)}\left(\mathbf{x}_{\mathbf{r}} ; \mathbf{y}_{\mathbf{r}}\right)},
$$

where

$$
\mathfrak{S}_{v(H o m)}\left(\mathbf{x}_{\mathbf{r}} ; \mathbf{y}_{\mathbf{r}}\right)=\prod_{\substack{i+j \leq n-2 \\ \alpha \leq r_{i i}, \beta \leq r_{n-j, n-j}}}\left(x_{\alpha}^{i}-y_{\beta}^{n-j}\right)
$$

It is an easy consequence of known facts about double Schubert polynomials (see, e.g., [18]) and the definition of $v(\mathbf{r})$ that $\mathfrak{S}_{v(H o m)}$ divides $\mathfrak{S}_{v(\mathbf{r})}$.

For an integer $m \geq 0$, let $m+\mathbf{r}$ be the set of rank conditions $\left\{m+r_{i j}\right\}$, for $0 \leq i \leq j \leq n$. It is shown that the limit

$$
F_{\mathbf{r}}(\mathbf{x}-\mathbf{y}):=\lim _{m \rightarrow \infty} Q_{m+\mathbf{r}}(\mathbf{x}-\mathbf{y})
$$

exists [24, Proposition 6.3]. That is, the coefficient of any fixed monomial eventually becomes constant.

Recall $W_{R P}(\mathbf{r})$ is the set of those $W \in W_{\min }(\mathbf{r})$ for which there is a $D \in \mathcal{R} C(v(\mathbf{r}))$ such that $\Phi(D)=W$. It is proved combinatorially that

$$
F_{\mathbf{r}}\left(\mathbf{x}_{\mathbf{r}}-\mathbf{y}_{\mathbf{r}}\right)=\sum_{W \in W_{R P}(\mathbf{r})} F_{w_{1}}\left(\mathbf{x}_{\mathbf{r}}^{0}-\mathbf{y}_{\mathbf{r}}^{1}\right) \cdots F_{w_{n}}\left(\mathbf{x}_{\mathbf{r}}^{n-1}-\mathbf{y}_{\mathbf{r}}^{n}\right),
$$

where $W=\left(w_{1}, \ldots, w_{n}\right)$.

There are two facts coming from geometry that are needed. The first is:

$$
\left[\Omega_{\mathbf{r}}\right]=Q_{\mathbf{r}}(\mathbf{x}-\check{\mathbf{x}})
$$


which is derived from an alternate form of a geometric construction originally due to Zelevinsky [32], and developed scheme-theoretically by Lakshmibai and Magyar [25] (and also reproved in [24]). The second is:

$$
c_{\underline{\mu}}(m+\mathbf{r})=c_{\underline{\mu}}(\mathbf{r})
$$

for all $\mu$ and $m \geq 0$, which is a consequence of the main theorem of [9].

By (11) and the main theorem of [9], one has

$$
Q_{\mathbf{r}}(\mathbf{x}-\check{\mathbf{x}})=\sum_{\underline{\mu}} c_{\underline{\mu}}(\mathbf{r}) s_{\mu_{1}}\left(\mathbf{x}_{\mathbf{r}}^{0}-\mathbf{x}_{\mathbf{r}}^{1}\right) \cdots s_{\mu_{n}}\left(\mathbf{x}_{\mathbf{r}}^{n-1}-\mathbf{x}_{\mathbf{r}}^{n}\right)
$$

Since this holds for any ranks $\mathbf{r}$, it holds for $m+\mathbf{r}$ when $m$ is large. By (9) and (12),

$$
F_{\mathbf{r}}(\mathbf{x}-\check{\mathbf{x}})=\sum_{\underline{\mu}} c_{\underline{\mu}}(\mathbf{r}) s_{\mu_{1}}\left(\mathbf{x}^{0}-\mathbf{x}^{1}\right) \cdots s_{\mu_{n}}\left(\mathbf{x}^{n-1}-\mathbf{x}^{n}\right) .
$$

Then (3) follows after specializing $\mathbf{x}^{i}$ to $\mathbf{x}_{\mathbf{r}}{ }^{i}$ for each $i$, i.e., by setting all "tail" variables $x_{j}^{i}$ for $j \geq r_{i i}+1$ to zero.

At this point, this argument gives a formula for $\left[\Omega_{\mathrm{r}}\left(E_{\mathbf{0}}\right)\right]$ as a multiplicity-free sum of products of Stanley functions over some minimal length lacing diagrams for $\mathbf{r}$. It remains to show that actually all appear. The proof of this fact in [24] was obtained from the geometric method of Gröbner degeneration, by subsequently applying multidegree formulae for matrix Schubert varieties from [23]. However, this is also immediate from Theorem 1. This completes a combinatorial derivation of (3) from the ratio formula (although we emphasize that the proof of the latter very much depends on geometry). Note that in this proof, facts coming from geometry are only required in order to connect the combinatorics of the polynomials above to quiver cycles.

In [9], an explicit positive combinatorial formula was conjectured for $c_{\mu}(\mathbf{r})$. This is proved in [24] using combinatorics, together with the ratio formula and the component formula. Thus, Theorem 1 also allows for a combinatorial proof of that conjecture, starting from the ratio formula.

\section{Splitting Schubert polynomials for classical Lie types}

In this section, we present "splitting" formulas for Schubert polynomials in each of the classical Lie types, i.e., formulas for polynomial representatives of Schubert classes in the cohomology ring of generalized flag varieties [3, 5]. In [10], a splitting formula for the Schubert polynomials of [27] was deduced from Theorem 4. Our analogues use the Schubert polynomials of types $B_{n}, C_{n}$ and $D_{n}$ defined by Billey and Haiman [4].

For a permutation $w \in S_{n}$ and a sequence of nonnegative integers $\left\{a_{j}\right\}$ with $1 \leq a_{1}<$ $a_{2}<\cdots<a_{k}<n$, we say that $w$ is compatible with $\left\{a_{j}\right\}$ if whenever $\ell\left(w s_{i}\right)<\ell(w)$ for a simple transposition $s_{i}$, then $i \in\left\{a_{j}\right\}$. Also, let $\operatorname{col}(T)$ denote the column word of a semi-standard Young tableau $T$, the word obtained by reading the entries of the columns of 


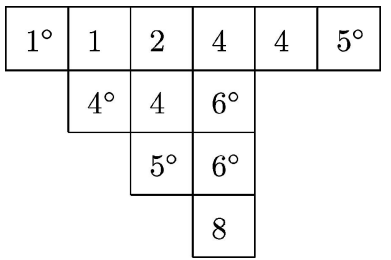

Figure 4. A circled shifted tableau for $\mu=(6>3>2>1)$.

the tableau from bottom to top and left to right. The following is the splitting formula for the $A_{n-1}$ Schubert polynomials of [27]:

Theorem 2 ([10]) Suppose $w \in S_{n}$ is compatible with $\left\{a_{1}<a_{2}<\cdots<a_{k}\right\}$. Then we have

$$
\mathfrak{S}_{w}(X)=\sum_{\underline{\lambda}} c_{\underline{\lambda}}(w) s_{\lambda^{1}}\left(X_{1}\right) \cdots s_{\lambda^{k}}\left(X_{k}\right)
$$

where $X_{i}=\left\{x_{a_{i-1}+1}, \ldots, x_{a_{i}}\right\}$ and the sum is over all sequences of partitions $\underline{\lambda}=\left(\lambda^{1}, \ldots\right.$, $\left.\lambda^{k}\right)$. Each $c_{\lambda}(w)$ is a quiver coefficient, equal to the number of sequences of semi-standard tableaux $\left(T_{1}, \ldots, T_{k}\right)$ such that:

(i) $T_{1}, T_{2}, \ldots, T_{k}$ have entries strictly larger than $0, a_{1}, \ldots, a_{k-1}$ respectively;

(ii) the shape of $T_{i}$ is conjugate to $\lambda^{i}$;

(iii) $\operatorname{col}\left(T_{1}\right) \cdots \operatorname{col}\left(T_{k}\right)$ is a reduced word for $w$.

We will need some notation and definitions. When $\mu=\left(\mu_{1}>\mu_{2}>\cdots>\mu_{\ell}\right)$ is a partition with $\ell$ distinct parts, there is a shifted shape given by a Ferrers shape of $\mu$ where each row is indented one space from the left of the row above it. A shifted tableau of shape $\mu$ is a filling of the shifted shape of $\mu$ by numbers and circled numbers $1^{\circ}<1<2^{\circ}<2<\cdots$ that is non-decreasing along each row and column. A shifted tableau is a circled shifted tableau if no circled number is repeated in any row and no uncircled number is repeated in any column, see e.g., figure 4 .

The weight $x^{T}=x_{1}^{w_{1}} x_{2}^{w_{2}} \ldots$ of a circled shifted tableau is defined by setting $w_{i}$ to be the number of $i$ or $i^{\circ}$ occurring in $T$. With this, the Schur $Q$ function $Q_{\mu}(X)$ is defined as $\sum_{T} x^{T}$, taken over all circled shifted tableaux of shape $\mu$. The Schur $P$ function $P_{\mu}(X)$ is defined to be $2^{-\ell(\mu)} Q_{\mu}(X)$, where $\ell(\mu)$ is the number of parts of $\mu$ (see, e.g., [20, 21]).

The Weyl group for the types $B_{n}$ and $C_{n}$ is the hyperoctahedral group $\mathbb{B}_{n}$ of signed permutations on $\{1,2, \ldots, n\}$. It is generated by the simple transpositions $s_{i}$ for $1 \leq i \leq$ $n-1$ together with the special generator $s_{0}$, which changes the sign of the first entry of the signed permutation. The Weyl group of type $D_{n}$ is the subgroup $\mathbb{D}_{n}$ of $\mathbb{B}_{n}$ whose elements make an even number of sign changes. It is generated by the simple transpositions $s_{i}$ for $1 \leq i \leq n-1$ together with $s_{\hat{0}}=s_{0} s_{1} s_{0}$. 
The $B_{n}$ and $D_{n}$ analogues of Stanley functions, $F_{w}(X)$ for $w \in \mathbb{B}_{n}$ and $E_{w}(X)$ for $w \in \mathbb{D}_{n}$, respectively, are defined in [4] by

$$
F_{w}(X)=\sum_{\mu} f_{w \mu} Q_{\mu}(X)
$$

and

$$
E_{w}(X)=\sum_{\mu} e_{w \mu} P_{\mu}(X)
$$

for certain nonnegative integers $f_{w \mu}$ and $e_{w \mu}$ given by explicit positive combinatorial formulas which we will not reproduce here; see [4] for details.

In [4], the theory of $A_{n-1}$ Schubert polynomials [27] was extended to types $B_{n}, C_{n}$ and $D_{n}$ (see [17] for an alternative approach). For types $B_{n}, C_{n}$ and $D_{n}$, the Schubert polynomials $\mathfrak{B}_{n}, \mathfrak{C}_{n}$ and $\mathfrak{D}_{n}$ respectively live in the polynomial ring $\mathbb{Q}\left[x_{1}, x_{2}, \ldots ; p_{1}(Z), p_{2}(Z), \ldots\right]$, where $p_{k}(Z)=z_{1}^{k}+z_{2}^{k}+\cdots$ is a power series in a new collection of variables $Z=$ $\left\{z_{1}, z_{2}, \ldots\right\}$. It is then proved in [4] that for $w \in \mathbb{B}_{n}$,

$$
\mathfrak{C}_{w}=\sum_{u, v} F_{u}(Z) \mathfrak{S}_{v}(X)
$$

where the sum is over $u \in \mathbb{B}_{n}$ and $v \in S_{n}$ with $u v=w$ and $\ell(u)+\ell(v)=\ell(w)$. Also, if $s(w)$ is the number of sign changes of $w$, then

$$
\mathfrak{B}_{w}=2^{-s(w)} \mathfrak{C}_{w} .
$$

Similarly for $w \in \mathbb{D}_{n}$,

$$
\mathfrak{D}_{w}=\sum_{u, v} E_{u}(Z) \mathfrak{S}_{v}(X)
$$

where the sum is over $u \in \mathbb{D}_{n}$ and $v \in S_{n}$, with $u v=w$ and $\ell(u)+\ell(v)=\ell(w)$.

More generally, if $w \in \mathbb{B}_{n}$ and a sequence of nonnegative integers $\left\{a_{j}\right\}$ with $1 \leq a_{1}<$ $a_{2}<\cdots<a_{k}<n$, we say that $w$ is compatible with $\left\{a_{j}\right\}$ if whenever $\ell\left(w s_{i}\right)<\ell(w)$ for a simple transposition $s_{i}$, then $i \in\left\{a_{j}\right\}$.

Theorem 3 Let $w \in \mathbb{B}_{n}$ be compatible with $\left\{a_{1}<a_{2}<\cdots<a_{k}\right\}$. Then we have

$$
\mathfrak{C}_{w}=\sum_{\mu ; \underline{\lambda}} c_{\mu ; \underline{\lambda}}(w) Q_{\mu}(Z) s_{\lambda^{1}}\left(X_{1}\right) s_{\lambda^{2}}\left(X_{2}\right) \cdots s_{\lambda^{k}}\left(X_{k}\right)
$$

and

$$
\mathfrak{B}_{w}=2^{-s(w)} \sum_{\mu ; \underline{\lambda}} c_{\mu ; \underline{\lambda}}(w) Q_{\mu}(Z) s_{\lambda^{1}}\left(X_{1}\right) s_{\lambda^{2}}\left(X_{2}\right) \cdots s_{\lambda^{k}}\left(X_{k}\right) .
$$


If in addition, $w \in \mathbb{D}_{n}$, then

$$
\mathfrak{D}_{w}=\sum_{\mu ; \underline{\underline{\lambda}}} d_{\mu ; \underline{\lambda}}(w) P_{\mu}(Z) s_{\lambda^{1}}\left(X_{1}\right) s_{\lambda^{2}}\left(X_{2}\right) \cdots s_{\lambda^{k}}\left(X_{k}\right)
$$

In the above formulas, $X_{i}=\left\{x_{a_{i-1}+1}, \ldots, x_{a_{i}}\right\}, \mu$ is a partition with distinct parts and $\underline{\lambda}=\left(\lambda^{1}, \ldots, \lambda^{k}\right)$ is a sequence of partitions. Also, $c_{\mu ; \lambda}(w)=f_{u \mu} c_{\lambda}(v)$ and $d_{\mu ; \lambda}=e_{u \mu} c_{\lambda}(v)$ where $u v=w, \ell(u)+\ell(v)=\ell(w), v \in S_{n}$, and $u \in \mathbb{B}_{n}$ or $u \in \mathbb{D}_{n}$, respectively.

Proof: Suppose $w \in \mathbb{B}_{n}$ (or respectively, $w \in \mathbb{D}_{n}$ ) and $u v=w$ with $\ell(u)+\ell(v)=\ell(w)$ where $u \in \mathbb{B}_{n}$ (or $u \in \mathbb{D}_{n}$ ) and $v \in S_{n}$.

Let $i \geq 1$ be such that $\ell\left(v s_{i}\right)<\ell(v)$. Then by our assumptions and standard properties of the length function (see, e.g., [22, Section 5.2]) we have

$$
\ell\left(w s_{i}\right)=\ell\left(u v s_{i}\right) \leq \ell(u)+\ell\left(v s_{i}\right)<\ell(u)+\ell(v)=\ell(w) .
$$

Hence $i$ is one of the $a_{j}$, i.e., $v$ is compatible with $\left\{a_{j}\right\}$. Therefore, the result follows from Eqs. (14), (15) and (16) combined with Theorem 2.

Example 5 Consider $w=\left(\begin{array}{rrr}1 & 2 & 3 \\ 3 & 1 & -2\end{array}\right)=s_{1} s_{0} s_{1} s_{2} s_{1} \in \mathbb{B}_{3}$. This signed permutation is compatible with the sequence $1<2$. In [4] the following was computed:

$$
\mathfrak{C}_{w}=Q_{41}+Q_{4} x_{1}+Q_{31} x_{1}+Q_{3} x_{1}^{2}+Q_{31} x_{2}+Q_{3} x_{1} x_{2}+Q_{21} x_{1} x_{2}+Q_{2} x_{1}^{2} x_{2} .
$$

This may be rewritten as

$$
\begin{aligned}
\mathfrak{C}_{w}= & Q_{41}+Q_{4} s_{1}\left(x_{1}\right)+Q_{31} s_{1}\left(x_{1}\right)+Q_{31} s_{1}\left(x_{2}\right)+Q_{3} s_{2}\left(x_{1}\right) \\
& +Q_{3} s_{1}\left(x_{1}\right) s_{1}\left(x_{2}\right)+Q_{21} s_{1}\left(x_{1}\right) s_{1}\left(x_{2}\right)+Q_{2} s_{2}\left(x_{1}\right) s_{1}\left(x_{2}\right)
\end{aligned}
$$

in agreement with Theorem 3.

In [10] it was explained why (13) provides a geometrically natural solution to the Giambelli problem for partial flag varieties. For the other classical types, the choice of variables makes it unclear what the underlying geometry of (17), (18) and (19) might be. On the other hand, given the shape of the formulas, by analogy with the $A_{n-1}$ case, it should be an interesting project to find a degeneracy locus setting for which the coefficients $c_{\mu ; \underline{\lambda}}(w)$ and $d_{\mu ; \underline{\lambda}}(w)$ (and their positivity) appear.

We also conjecture that these numbers can also be naturally realized as Schubert structure constants of the corresponding Lie type, in analogy with [12]. In fact, one of the bizarre twists in this story, as explained in [12], is that while the type $A$ splitting coefficients are all special cases of the general quiver coefficients, the opposite is true (in a natural way) also! We expect that the eventual degeneracy locus setting we seek for the other classical types should exhibit such relations as well. 


\section{Appendix: Relations to Fulton's universal Schubert polynomials}

In this Appendix, we report on the details of a bijection which shows how the component formula (3) generalizes a formula for Fulton's universal Schubert polynomials given in [10]. This bijection was also found independently in [24], where a proof was sketched. We provide another proof below.

Let $\mathfrak{X}$ be a nonsingular complex variety and let

$$
G_{1} \rightarrow \cdots \rightarrow G_{n-1} \rightarrow G_{n} \rightarrow H_{n} \rightarrow H_{n-1} \rightarrow \cdots \rightarrow H_{1}
$$

be a sequence of vector bundles and morphisms over $\mathfrak{X}$, such that $G_{i}$ and $H_{i}$ have rank $i$ for each $i$. For every permutation $w$ in the symmetric group $S_{n+1}$ there is a degeneracy locus

$$
\Omega_{w}\left(G_{\bullet} \rightarrow H_{\bullet}\right)=\left\{x \in \mathfrak{X} \mid \operatorname{rank}\left(G_{q}(x) \rightarrow H_{p}(x)\right) \leqslant r_{w}(p, q) \text { for all } 1 \leqslant \mathrm{p}, \mathrm{q} \leqslant \mathrm{n}\right\},
$$

where $r_{w}(p, q)$ is the number of $i \leqslant p$ such that $w(i) \leqslant q$. The universal double Schubert polynomial $\mathfrak{S}_{w}(c ; d)$ of Fulton [19] gives a formula for this locus; this is a polynomial in the Chern classes $c_{i}(j)=c_{i}\left(H_{j}\right)$ and $d_{i}(j)=c_{i}\left(G_{j}\right)$ for $1 \leqslant i \leqslant j \leqslant n$. These polynomials are known to specialize to the single and double Schubert polynomials and the quantum Schubert polynomials $[13,15]$.

The loci associated with universal Schubert polynomials are special cases of these quiver cycles. Given $w \in S_{n+1}$ we define rank conditions $\mathbf{r}^{(n)}(w)=\left\{r_{i j}^{(n)}\right\}$ for $1 \leqslant i \leqslant j \leqslant 2 n$ by

$$
r_{i j}^{(n)}= \begin{cases}r_{w}(2 n+1-j, i) & \text { if } i \leqslant n<j \\ i & \text { if } j \leqslant n \\ 2 n+1-j & \text { if } i \geqslant n+1\end{cases}
$$

The expected (and maximal) codimension of this locus is $\ell(w)$.

Thus the quiver polynomial specializes to give a formula for the universal Schubert polynomial. We say that a product $u_{1} \cdots u_{2 n-1}$ is a reduced factorization of $w$ if $u_{1} \cdots u_{2 n-1}=w$ and $\ell\left(u_{1}\right)+\cdots+\ell\left(u_{2 n-1}\right)=\ell(w)$. The following was proved ${ }^{2}$ :

Theorem 4 ([10]) For $w \in S_{n+1}$,

$$
\Omega_{\mathbf{r}^{(n)}(w)}=\sum_{u_{1} u_{2} \cdots u_{2 n-1}=w} F_{u_{1}}\left(G_{1}-G_{2}\right) \cdots F_{u_{2 n-1}}\left(H_{2}-H_{1}\right)
$$

where the sum is over all reduced factorizations $w=u_{1} \cdots u_{2 n-1}$ such that $u_{i} \in S_{\min (i, 2 n-i)}$ +1 for each $i$.

There does not appear to be any a priori reason, such as by linear independence or geometry, that proves that this expansion coincides with (3) under the conditions (21) and (22). However, this follows from: 
Proposition 3 The map $\Gamma$ that sends $W=\left(w_{1}, \ldots, w_{2 n-1}\right) \in W_{\min }\left(\mathbf{r}_{w}^{(n)}\right)$ to $\tilde{w}_{2 n-1}^{-1} \tilde{w}_{2 n-2}^{-1} \ldots$ $\tilde{w}_{1}^{-1}$ is a bijection between minimal length lacing diagrams of $\mathbf{r}_{w}^{(n)}$ and reduced factorizations of $w=u_{1} \cdots u_{2 n-1}$ such that $u_{i} \in S_{\min (i, 2 n-i)+1}$ for each $i$.

Example 6 Let $n=2$ and $w=s_{2} s_{1}=\left(\begin{array}{lll}1 & 2 & 3 \\ 3 & 1 & 2\end{array}\right) \in S_{3}$. This corresponds to the following rank conditions:

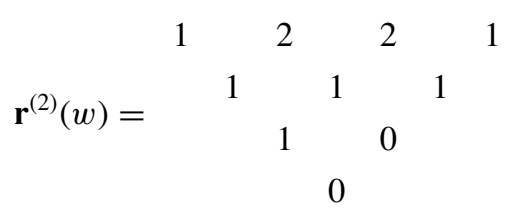

Recall the definition of lacing diagrams from pg. 4. The unique lacing diagram associated to $\mathbf{r}^{(2)}(w)$ is drawn below with bold lines and solid vertices. By drawing "phantom" laces and vertices, $w$ is encoded by reading the paths from right-to-left.

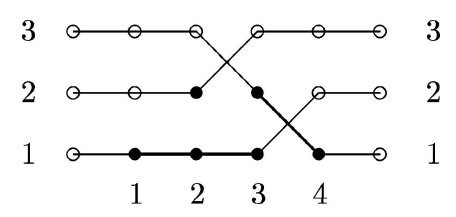

Proof of Proposition 3: The following lemma is an easy consequence of the definition of $r_{w}(p, q)$ :

Lemma 3 Let $w \in S_{n+1}$, then $r_{w}(p, q)-r_{w}(p-1, q)-r_{w}(p, q-1)+r_{w}(p-1, q-1)$ is equal to 1 if $w(p)=q$ and is equal to 0 otherwise. Here we $\operatorname{set}_{w}(p, q)=0$ if $p<0$ or $q<0$.

Lemma 3 combined with (4) and (22) implies that $s_{i j}\left(\mathbf{r}_{w}^{(n)}\right)$ for $1 \leq i \leq j \leq 2 n$ is 1 if $(i, j)$ falls into one of the following three cases:

(i) $(w(\alpha), 2 n-\alpha+1)$ and $1 \leq w(\alpha) \leq n, 1 \leq \alpha \leq n$;

(ii) $(w(n+1), n)$ and $w(n+1) \neq n+1$;

(iii) $\left(n+1,2 n-w^{-1}(n+1)+1\right)$ and $w^{-1}(n+1) \neq n+1$;

and is equal to 0 otherwise.

First, we check that $\Gamma$ is well-defined. If $W=\left(w_{1}, w_{2}, \ldots, w_{2 n-1}\right) \in W_{\min }\left(\mathbf{r}_{w}^{(n)}\right)$ then it is immediate from (22) that $\tilde{w}_{2 n-i}^{-1} \in S_{\min (i, 2 n-i)+1}$ for $1 \leq i \leq 2 n-1$. Also the conditions (i), (ii) and (iii) are exactly saying that $\tilde{w}_{2 n-1}^{-1} \tilde{w}_{n-1}^{-1} \cdots \tilde{w}_{1}^{-1}=w$ (e.g., by generalizing the 
picture in Example 6). Further, since

$$
\ell\left(\tilde{w}_{2 n-1}^{-1}\right)+\cdots+\ell\left(\tilde{w}_{1}^{-1}\right)=d\left(\mathbf{r}_{w}^{(n)}\right)=l(w),
$$

this factorization of $w$ is reduced.

It is clear that $\Gamma$ is injective. To check surjectivity, let $u_{1} u_{2} \cdots u_{2 n-1}$ be a reduced factorization of $w$ such that $u_{i} \in S_{\min (i, 2 n-i)+1}$. Then let $W=\left(u_{2 n-1}, \ldots, u_{1}\right)$ be the lacing diagram obtained by interpreting each $u_{2 n-i}$ as the partial permutation represented by a $\min (i, 2 n-i) \times(\min (i, 2 n-i)+1)$ matrix, for $i<n$ and $(\min (i, 2 n-i)+1) \times \min (i, 2 n-i)$ matrix for $i>n$, and an $n \times n$ matrix for $i=n$ (in the last case, we ignore $n+1$ in the domain and range of $u_{n}$ ). This combined with $u_{1} \cdots u_{2 n-1}=w$ shows there is a unique $(i, j)$-lace when one of the conditions (i), (ii) or (iii) hold, and no other laces. Thus our calculation of $s\left(\mathbf{r}_{w}^{(n)}\right)$ shows $W$ is a lacing diagram for $\mathbf{r}_{w}^{(n)}$. This lacing diagram is of minimal length since $u_{1} u_{2} \cdots u_{2 n-1}=w$ is a reduced factorization and $\ell(w)=d\left(\mathbf{r}_{w}^{(n)}\right)$. Finally, $\Gamma$ maps $W$ to $u_{1} u_{2} \cdots u_{2 n-1}$, as desired.

\section{Notes}

1. Unfortunately, a pipe network for $w$ is not the same as a pipe dream for $w$. In the literature, the " $w$ " in the latter would refer to the Demazure product of the corresponding Hecke word determined by the pipes, not where pipes exit from.

2. See also [11] for a $K$-theoretic generalization.

\section{References}

1. S. Abeasis and A. Del Fra, "Degenerations for the representations of an equioriented quiver of type $A_{m}$," Boll. Un. Mat. Ital. Suppl. 2 (1980), 157-171.

2. N. Bergeron and S. Billey, "RC-graphs and Schubert polynomials," Experimental Math. 2(4) (1993), 257-269.

3. I.N. Berstein, I.M. Gelfand, and S.I. Gelfand, "Schubert cells and cohomology of the spaces $G / P$," Russian Math. Surveys 28 (1973), 1-26.

4. S. Billey and M. Haiman, "Schubert polynomials for the classical groups," J. Amer. Math. Soc. 8 (1995), $443-482$.

5. A. Borel, "Sur la cohomologie des espaces fibrés principaux et des espaces homogènes de groupes de Lie compacts," Ann. of Math. 57 (1953), 115-207.

6. A.S. Buch, "Stanley symmetric functions and quiver varieties," J. Algebra 235 (2001), 243-260.

7. A.S. Buch, "Grothendieck classes of quiver varieties," Duke Math. J. 115(1) (2002), 75-103.

8. A.S. Buch, "Alternating signs of quiver coefficients" (preprint).

9. A.S. Buch and W. Fulton, "Chern class formulas for quiver varieties," Invent. Math. 135 (1999), $665-687$.

10. A.S. Buch, A. Kresch, H. Tamvakis, and A. Yong, "Schubert polynomials and quiver formulas," Duke Math. J. 122(1) (2004), 125-143.

11. A.S. Buch, A. Kresch, H. Tamvakis, and A. Yong, "Grothendieck polynomials and quiver formulas," Amer. J. Math. (to appear).

12. A.S. Buch, F. Sottile, and A. Yong, "Quiver coefficients are Schubert structure constants," (preprint).

13. I. Ciocan-Fontanine, "On quantum cohomology rings of partial flag varieties," Duke Math. J. 98(3) (1999), 485-524.

14. M. Edelman and C. Greene, "Balanced tableaux," Adv. Math. 63 (1987), 42-99.

15. S. Fomin, S. Gelfand, and A. Postnikov, "Quantum Schubert polynomials," J. Amer. Math. Soc. 10 (1997), $565-596$. 
16. S. Fomin and C. Greene, "Noncommutative Schur functions and their applications," Discrete Math. 193(1-3) (1998), 179-200.

17. S. Fomin and A.N. Kirillov, "Combinatorial $B_{n}$-analogues of Schubert polynomials," Trans. Amer. Math. Soc. 348(9) (1996), 3591-3620.

18. S. Fomin and A.N. Kirillov, "The Yang-Baxter equation, symmetric functions, and Schubert polynomials," Discrete Math. 153(1-3) (1996), 123-143, Proceedings of the 5th Conference on Formal Power Series and Algebraic Combinatorics (Florence, 1993).

19. W. Fulton, “Universal Schubert polynomials,” Duke Math. J. 96(3) (1999), 575-594.

20. W. Fulton and P. Pragacz, Schubert Varieties and Degeneracy Loci, Lecture Notes in Mathematics, Vol. 1689, Springer-Verlag, New York, 1998.

21. P. Hoffman and J. Humphreys, Projective Representations of the Symmetric Groups. Q-Functions and Shifted Tableaux, The Clarendon Press, Oxford University Press, New York, 1992.

22. J. Humphreys, Reflection Groups and Coxeter Groups, Cambridge University Press, Cambridge, 1990.

23. A. Knutson and E. Miller, "Gröbner geometry of Schubert polynomials," Ann. of Math. (to appear).

24. A. Knutson, E. Miller, and M. Shimozono, "Four positive formulae for type A quiver polynomials," (preprint).

25. V. Lakshmibai and P. Magyar, "Degeneracy schemes, quiver schemes, and Schubert varieties," Internat. Math. Res. Notices 12 (1998), 627-640.

26. A. Lascoux, "Classes de Chern des variétès de drapeaux," C.R. Acad. Sci. Paris Sér. I Math. 295(5) (1982), 393-398.

27. A. Lascoux and M.-P. Schützenberger, "Polynômes de Schubert," C.R. Acad. Sci. Paris Sér. I Math. 294 (1982), 447-450

28. A. Lascoux and M.-P. Schützenberger, "Structure de Hopf de l'anneau de cohomologie et de l'anneau de Grothendieck d'une variété de drapeaux," C.R. Acad. Sci. Paris Sér. I Math. 295 (1982), 629-633.

29. I.G. Macdonald, Notes on Schubert Polynomials, Publ. LACIM 6, Univ. de Québec à Montréal, Montréal, 1991.

30. L. Manivel, Symmetric Functions, Schubert Polynomials and Degeneracy Loci, American Mathematical Society, Providence 2001

31. E. Miller, "Alternating formulae for $K$-theoretic quiver polynomials," Duke Math. J. (to appear).

32. A.Zelevinsky, "Two remarks on graded nilpotent classes," Uspehi. Mat. Nauk. 40 (1985) vol. 1 (241), 199-200. 